\title{
Stopped hearts, amputated toes and NASA: contemporary legends among healthy volunteers in US phase I clinical trials
}

\author{
Jill A. Fisher \\ Department of Social Medicine and Center for Bioethics, University of North Carolina, North \\ Carolina, USA
}

\section{Abstract}

The first stage of testing new pharmaceuticals in humans is referred to as a phase I clinical trial. The purpose of these studies is to test the safety of the drugs and to establish appropriate doses that can later be given to patients. Most of these studies are conducted under controlled, in-patient conditions using healthy volunteers who are paid for their participation. To explore healthy volunteers' experiences in clinical trials, an ethnographic study was conducted at six in-patient phase I clinics in the USA. In addition to the observation of clinic activities (from informed consent procedures to dosing to blood draws), 268 semi-structured interviews were conducted, 33 with clinic staff and 235 with healthy volunteers. Drawing on this dataset, this article explores healthy volunteers' exchange of contemporary legends about phase I clinical trials. In addition to potentially scaring the listener and communicating distrust in the medical community, these incredible stories help participants cope with perceived stigma and establish a gradient of risk of trial participation, creating potential boundaries to their participation in medical research. The article argues that contemporary legends play a productive role in society, shaping how people view themselves and others and influencing their decisions about risky activities.

\section{Keywords}

clinical trials; phase I; safety; contemporary legends; healthy volunteers; pharmaceuticals

\begin{abstract}
Throughout the USA, if one asks healthy volunteers in pharmaceutical clinical trials about the riskiest or most invasive medical studies they have heard about, the same contemporary legends are likely to be told and retold. Stories abound about researchers paralysing participants, putting participants into cardiac arrest, amputating their toes or thumbs and confining them to bed for months on end. There are also stories about dishonest participants whose lies about their medical histories or their past research experiences have led to their deaths. While others have noted the circulation of such tales as humourous folklore (for example, Abadie 2010, Helms 2005), I was struck during my ethnographic research by the importance of these stories for healthy volunteers. Forming part of the clinical culture of pharmaceutical trials, contemporary legends contribute to healthy volunteers' identities as
\end{abstract}

\section{(C) 2015 The Author.}

Address for correspondence: Jill A. Fisher Assistant Professor of Social Medicine, Center for Bioethics, University of North Carolina at Chapel Hill, 333 MacNider Hall, Campus Box 7240, 333 S. Columbia Road, Chapel Hill, NC 27599-7240, USA. jill.fisher@unc.edu. 
research participants and provide an outlet to process the stigma of and their fears about the risks of medical research.

Researchers use healthy volunteers in what are called phase I clinical trials to determine the safety of new products by measuring the absorption, metabolism, and excretion of these drugs in individuals with normal kidney and liver function and by observing any clinical effects (that is, symptoms) experienced by the participants (Corrigan, 2002). ${ }^{1}$ These studies are very different from many other clinical trials in that they usually include a confinement period during which participants must stay in the research clinic to be dosed with the investigational drug and have all required medical procedures, as well as sleep, have meals, and interact with other study participants (Fisher 2009). The confinement period creates the opportunity for the healthy volunteers to exchange stories about their own or others' experiences in previous phase I trials.

Participation in USA medical research is a stigmatised activity for many healthy volunteers, especially participants of minority groups (Corbie-Smith et al. 1999, Shavers-Hornaday et al. 1997). In spite of scholarly concern about the low number of participants from minority groups in medical research, phase I clinical trials enrol an overrepresentation of both African American and Hispanic healthy volunteers (Fisher and Kalbaugh 2011). Regardless of the perceived stigma, healthy volunteers from all racial and ethnic backgrounds are primarily motivated by the financial compensation. Indeed, the amount of compensation (up to $\$ 7000$ per study) attracts many healthy volunteers to participate serially in phase I studies, and repeat participants predominate in phase I clinics (Almeida et al. 2007, Tishler and Bartholomae 2003). Some participants even consider themselves professional research subjects and earn their income exclusively from phase I clinical trials (Abadie 2010, Elliott 2008).

This article examines one key aspect of the interactions among healthy volunteers brought together by their common interest in the money they can gain from phase I trials: the exchange of contemporary legends about medical research. These stories are particularly interesting narratives because, in contrast to the volunteers' actual experiences of phase I trials, the legends tend to portray these studies as extraordinarily invasive, dangerous and possibly illegitimate, and as offering enormous sums of money for taking these risks. Drawing upon an ethnographic study of six US phase I clinics, I illustrate how the circulation of contemporary legends helps participants cope with the stigma of participating in medical research and establishes a gradient of study risk, creating potential boundaries for their participation in phase I trials. I argue that contemporary legends play a productive role in society, shaping how people view themselves and others and influencing their decisions about risky activities.

\footnotetext{
${ }^{1}$ Most countries' drug regulatory agencies require that pharmaceuticals must be tested for safety and efficacy (Donohue 2006). Phase I studies are designed solely to test safety. Phase II studies usually recruit patients in order to collect additional information about safety in the affected population as well as to provide preliminary information about efficacy. Phase III studies are larger scale efficacy studies on patients designed to show that an investigational drug is either better than a placebo or approved drugs.
} 


\section{Literature on contemporary legends}

In the early 1980s there was a resurgence of interest in sociology in contemporary legends, as folklore studies' scholar Jan Brunvand $(1981,1984)$ published his first two popular books on the topic. The field of sociology had a long-standing interest in rumour, especially as a phenomenon of collective behaviour (Addams 1914, Shibutani 1966), but the two types of stories differ in important ways. Rumours can be thought of as unverified information statements that arise from ambiguous or threatening situations (DeFonzo and Bordia 2007). They also tend to be a twist or embellishment on the everyday and are anchored in time to a specific person, place or context. In contrast to rumours, contemporary legends, or more colloquially, urban legends or myths, are stories with longevity that circulate widely and are told as true events that occurred to someone within the teller's social network but who is not directly known to the teller. Using elements of humour as well as horror, the stories tend to contain a moral, often communicating a warning to the listener (DiFonzo and Bordia 2007). For example,Joel Best and Gerald Horiuchi (1985) analysed the 1970s emergence of contemporary legends about Halloween sadists, individuals who were adulterating candy to kill or harm children. By investigating the real events that sparked widespread concern throughout the USA, Best and Horiuchi show that the vulnerability of children and distrust of strangers are critical themes in these stories. Indeed, vulnerability and distrust are common features of contemporary legends more generally (Fine 1992, Turner 1993). Similarly, Jeffrey Victor (1998) argues that the telling and retelling of contemporary legends is a form of collective behaviour that allows moral-political messages to take different shape over time to suit the needs of the narrative context. Interestingly, in spite of their often fantastic nature, the repetition and multiple exposure to contemporary legends make them seem more true to listeners (Fox Tree and Weldon 2007).

The medical profession is not immune to the circulation of contemporary legends. For example, Robert Dingwall (2001) illustrates how stories about kidney theft circulated in Nottingham, UK. An important characteristic of this contemporary legend is that the stories included specific details such as actual nightclubs where the victims were allegedly targeted. Dingwall shows how situating these details in specific identifiable locales adds credibility to the tales. Likewise, Véronique Campion-Vincent (2002) illustrates that global narratives about organ theft reveal a general mistrust of the medical profession, especially by the poor and those in developing countries. Campion-Vincent links these contemporary legends with similar anxieties about tissue transplantation and the creation of monsters by scientists, which are themes that have long been voiced in fiction, such as in Shelley's Frankenstein (1818) and Wells' The Island of Dr Moreau (1896).

What many contemporary legends have in common is that they are tales of risk, not just vulnerability and distrust. In the case of organ theft, risk is a central feature of the stories. Other contemporary legends, when circulated among a subgroup or subculture, can even be viewed as a method of communicating risk such that individuals change their behaviour. In a study of drug-using sex workers, for example, Brenda Roche et al. (2005) detail the ways in which contemporary legends can put women at greater risk (for example, by creating false information about risk or serving to rationalise behaviour) but also help prepare them for terrible incidents that can happen on the street, encouraging them to exercise greater caution. 
By positioning contemporary legends in this way, I see these tales as having productive functions. Rather than being told simply to scare or entertain the listener, contemporary legends can actively shape people's identities, perceptions, and behaviour. The current literature hints at this. Dingwall (2001) underscores the importance of attending to who recounts contemporary legends to whom and why. In his own research Dingwall finds that storytelling is a form of occupational boundary-maintenance, where having the right to tell certain stories defines healthcare workers' membership in their occupational group. Another revealing example of how the contemporary legend contributes to the formation of identities is described by Elaine Moriarty (2005) in her exploration of a popular Irish contemporary legend. She found that stories about immigrant women who abandoned baby carriages when boarding Dublin buses served to differentiate native-born Irish women from non-national immigrants, constructing the former as struggling citizens and the latter as ingrates who benefit from state handouts.

Few studies, however, have examined the productive functions of contemporary legends. In the case of risky or stigmatised activities in particular, what purpose do contemporary legends serve? Are they simply cautionary tales? Or do they help individuals make decisions about their actions? The case of phase I clinical trials is instructive in this regard. As will become clear, contemporary legends about phase I clinical trials exhibit to some degree all the characteristics detailed in the scholarly literature. Healthy volunteers pass on incredible stories that can scare the listener, manifest distrust in medical practitioners and form the basis of a shared community. Additionally, by establishing a fictionalised gradient of risk in medical research, contemporary legends help shift the stigma of participation while setting boundaries for the decisions healthy volunteers make about enrolling in clinical trials.

\section{Methods}

This article reports on an ethnographic study of six USA phase I facilities that I visited from November 2009 to October $2010 .^{2}$ The facilities were distributed across the USA, with two clinics in the East, two in the Midwest and two in the West. Five were commercial research clinics and one was part of an academic medical centre. The capacity of the clinics ranged from 16 to 300 beds (median $=51$ ). All facilities conducted phase I clinical trials exclusively.

My time in each facility varied, based on the facilities' schedules (that is, when they had active studies) as well as other local differences (for example, the research staff's comfort with being observed). In sum, I spent 38 days and about 450 hours in the six clinics, with my shortest stay lasting two days and the longest 14 days. On a typical day of research, I arrived at the clinic at $8 \mathrm{am}$ and concluded at $8 \mathrm{pm}$. On mornings with important dosing protocols or procedures, I arrived at the clinic by $6 \mathrm{am}$. Some days I stayed until $10 \mathrm{pm}$ to observe all the events of the day or use the time to conduct interviews during quiet moments in the clinic. As is typical with ethnographic methods, I simply made myself available while I was in the field and I adapted to the schedules of the facilities.

\footnotetext{
${ }^{2}$ I provide more complete findings from this broader ethnographic project in a book manuscript I am currently completing.
} 
My observational research consisted of both formal and informal strategies. Formal observations included sitting in on informed consent and screening processes, watching healthy volunteers receive drug doses and medical procedures, and listening to the staff handoff information at shift changes. The informal portion of my observations included downtime in the clinics when few structured activities occurred. During these times I often simply sat with healthy volunteers while they ate meals, played games and watched television. At other times I focused on the research staff, joining them in the nurses' station or in break rooms. During all my observations, I had countless conversations with staff and healthy volunteers in which they candidly told me about their experiences working in or participating in phase I trials.

I conducted a total of 268 semi-structured interviews across the six facilities. In all, 33 interviews were with research staff and included administrators, physicians (investigators), nurses, phlebotomists, project managers and recruiters. ${ }^{3}$ Interview questions were adapted to tap into each staff member's expertise and job responsibilities, but they were all asked about their perceptions of the healthy volunteers, including questions about how well informed they thought volunteers were about phase I trials. The interviews with the research staff ranged from 10 to 105 minutes, with an average of 44 minutes per interview.

I interviewed 235 healthy volunteers who at the time of the interview were either enrolled in or being screened for a study. ${ }^{4}$ The semi-structured interview guide was designed to solicit information about volunteers' experiences in phase I clinical trials, their motivations to enrol in studies, their perceptions of the risks and benefits and their willingness to participate in different types of clinical trials. My sample of healthy volunteers was quite diverse, accurately representing the population of healthy volunteers. They had a wide range of experience in participating in phase I clinical trials (number of trials: 1 to $100+$, mean $=7$, median $=3$ ). Healthy volunteers are predominantly male, and my sample reflected this trend with $73 \%$ of my sample being men. While the racial and ethnic backgrounds of participants varied by clinic and region of the USA (see Table 1), my overall sample of healthy volunteers included 37\% non-Hispanic white, 35\% African American, 22\% Hispanic, 4\% Asian and $1 \%$ Native American participants. Interviews with healthy volunteers ranged from five to 100 minutes, with an average of 17 minutes per interview. The length of the interviews with healthy volunteers varied in part because the duration was controlled by the participant but also because individuals who were relatively new to clinical trials had fewer examples and experiences to report.

The interviews were transcribed in full, imported to Atlas.ti with all my ethnographic field notes and coded. I used a multi-staged approach to coding, concentrating initially on broad themes (for example, risk, informed consent, motivation, adverse events and public perceptions of trials) then adding more nuanced codes in subsequent passes through the dataset (for example, rumours/urban legends, social network, selectivity: studies, selectivity: sites, gender dynamics, race/ethnicity). The later codes generally reflected unanticipated

\footnotetext{
${ }^{3}$ My sample of 33 research staff included 21 women and 12 men, of whom 25 were non-Hispanic whites, five African Americans, one Asian American, one Hispanic and one Native American.

${ }^{4}$ I conducted most of the interviews myself, but two graduate students conducted 28 interviews in Spanish with healthy volunteers at two clinics.
} 
categories - such as the presence and role of contemporary legends - that emerged from the data. The identities of phase I clinics and staff are confidential, and all the healthy volunteers are anonymous. The Vanderbilt University institutional review board reviewed and approved the research protocol.

\section{Contemporary legends about phase I participation}

Although I did not set out to study the stories that healthy volunteers tell about phase I trials, my interest in contemporary legends was piqued during my informal observations of healthy volunteers. Conversations among healthy volunteers are replete with brief, tactical information exchanges about upcoming studies, facilities that someone might want to consider trying or avoiding, and other snippets of information to help others participate in clinical trials. I observed that participants were especially likely to swap their more detailed study stories during meals, and invariably people's personal experiences in trials alternated with retellings of (often) outrageous contemporary legends about clinical trials in which a friend-of-a-friend participated.

Healthy volunteers are eager to share stories because in a typical clinical trial, most days are spent confined to a research facility with few scheduled events (for example, dosing, blood draws, electrocardiograms) to occupy their day. Indeed, many healthy volunteers remarked to me how boring clinical trials could be. Socialising with other volunteers is a diversion to help the time pass more quickly. Additionally, these conversations about clinical trials were an important outlet for the healthy volunteers. Many were hesitant to talk about their participation with anyone outside the studies because of the stigma associated with the activity (more on this below). In this context, the healthy volunteers' narrative transactions about phase I trials are critical in shaping individuals' perceptions of participation in such studies.

Contemporary legends are analytically interesting stories because listeners know better than to believe that the stories can possibly be true but the narratives nonetheless resonate with them to such an extent that they have trouble dismissing them outright as false. As with other contemporary legends, there is a strong moral contained in the stories in spite of variations in the details. Specifically, phase I contemporary legends tend to highlight risks of study participation, establishing a fictionalised gradient of risk to which volunteers can compare their current studies. I frequently heard versions of five phase I contemporary legends: spinal tap paralysis, the flatliner study, the amputated toe, the National Aeronautics and Space Administration (NASA) study and the dead participant. As I retell these stories in the sections that follow, I draw upon my data both to illustrate how healthy volunteers discuss these stories and to analyse the meaning of these tales. As I will show, the themes that emerge from the data about risk and financial desperation suggest that sharing contemporary legends has a productive function for healthy volunteers.

\section{Spinal tap paralysis}

Lumbar punctures are a relatively uncommon study procedure, but healthy volunteers discuss them as though they are widely used in clinical trials. The reason a protocol would include a lumbar puncture is to measure the degree to which an investigational drug crosses 
the blood-brain barrier, as evinced by its presence in cerebrospinal fluid (Cavens and Ramael 2009). Like many healthcare providers and patients, healthy volunteers generally use the informal label 'spinal tap' to refer to lumbar punctures, and these are one procedure that many participants claim they would never consent to as part of a clinical trial. These studies, however, tend to pay higher stipends, making them tempting enough to often put the decision against participation in question.

With so much fear of lumbar punctures, the healthy volunteers repeat elaborate tales about trial participants who were paralysed by this procedure. The basic narrative is simple: a participant enters a clinical trial that requires a lumbar puncture, the physician makes a mistake in inserting the needle and the participant leaves the clinic in a wheelchair and is paralysed for life. Some of the variations on the theme are interesting in spite of the standard storyline. For example, when one serial participant; an African American in his forties, told me his version of the contemporary legend, he made himself a personal witness to the events, which he situated in the phase I clinic where I interviewed him. He also personalised the participant by giving her a name and a vivid personality: 'Her name's Judith. She was a pool shark. She was killing everybody on the pool table. Older lady'. To conclude the story, the participant inserted himself into it directly by telling me:

She was in one of these rooms. They pulled her out in a wheelchair ... She said, 'Yeah, I'd do it again'. I said, 'Judith, for real? You can't walk now. Are you serious?' I wish I had that on YouTube.

As he relates this part of the story, the participant is laughing, and his tone simultaneously conveys criticism of and admiration for Judith. The context for telling me about Judith, was thinking about the lumbar puncture the participant personally had experienced, which gave him a colossal headache after getting out of bed too soon afterwards. Immediately before launching into the story, he was struggling to justify his enrolment in the lumbar puncture study:

Once you've got the $\$ 6,500$ check stuck in your mind, people are scared to get up and leave. [Laughs]. I was like, 'Spinal tap?! Look how much they're paying though'. It was like, it's the money.

Beyond the humour in his version of the contemporary legend, this participant was communicating a serious message about the influence of money on decisions about phase I trials.

\section{The 'flatliner' study}

The flatliner study is one of the most fascinating legends because it expresses the underlying fear that many healthy volunteers have about medical research and also the degree to which individuals' desperation can drive them to earn income in dangerous ways. As with the 1990 film thriller Flatliners, where medical students took turns stopping their hearts (that is, flatlining) to explore what exists after life, this phase I legend involves a heart study in which, for a substantial stipend, participants consent to allow the medical team to put them in cardiac arrest for a brief period of time, then resuscitate them. The period of time varies from 60 seconds to several minutes, and as healthy volunteers retell this contemporary 
legend, they ruminate on the length of time the participant is 'dead', implying that a shorter period of time makes the study more legitimate. The healthy volunteers seem quite conflicted about the veracity of this contemporary legend, knowing how unlikely it is to be true but unable to disregard it as fiction. For example, an African American serial participant in his thirties commented:

I don't know if it's a myth going around or something that actually happened. I heard a story about the university that stops your heart for 60 seconds or whatever and they pay you, I don't know, $\$ 50,000$ or whatever. I don't know anybody that's ever done it. [Laughs]. I just hear everybody talking about it. Maybe nobody ever lived to come back and talk about it.

It is the large stipend associated with the study that keeps the contemporary legend circulating. Healthy volunteers are intrigued by the possibility of such a huge study pay-out, and their financial need can add to their interest in it. Belief that the flatliner study exists even encourages some healthy volunteers to ask research staff how they can find and enrol in it. For example, during an interview with a phase I investigator, I asked him about his awareness of the contemporary legends that circulate among healthy volunteers. Immediately, he described his interaction with participants who ask him about the flatliner study:

So usually the context of the question is something like, 'Well, you know this $\$ 2,000, \$ 3,000$ study is nice, but I really need like 20 or 50 or 100,000 . I heard that there's this $\$ 100,000$ study out there that if you let the unit stop your heart, ... you'll get paid like 100,000 , so I'm really wanting that study... They really think that there's someone out there doing such crazy studies like that, and they can get paid a lot.

By perceiving the flatliner contemporary legend as true, healthy volunteers illustrate the extreme degree to which they normalise research risks and will consider endangering themselves if the price is right.

\section{The amputated toe}

As a strange twist on the flatliner study, a companion contemporary legend circulates about researchers who pay participants to have a toe amputated then - in nearly all the versions of the tale - reattached. Like the other story, participants are paid a large stipend to undergo these procedures, but it is unclear why the researchers have an interest in amputations or what the research goals could be. While I was in the field I also heard variations on the theme in which, instead of a toe, it was a finger or a large patch of skin that was subject to the procedures. When the healthy volunteers told me these contemporary legends, their tone was often more comedic, almost challenging the story even as they recounted it. After telling me detailed versions of the spinal tap and flatliner legends, an African American serial participant in his late thirties said:

You start hearing all the different stories [in the clinic] ... [Laughs] You hear some of the outrageous stuff. 'Man, they wanna cut off your thumbs' [Laughs] 'and your big toes and switch 'em around and reattach 'em. You know instead of a thumb 
he'll have a big toe [on his hand] for a couple of months and then they gonna put 'em back. They pay you $\$ 80,000$, man'.

This recounting of the way healthy volunteers tell each other these stories hints at some of the angst about medical research, channelling perhaps a tepid version of The Island of $\mathrm{Dr}$ Moreau, where the titular character creates monstrous animal hybrids through vivisection.

Compared to other contemporary legends, the healthy volunteers exhibited more incredulity about the amputated toe tale and many seemed to fixate on the nonsensical nature of the story. At one clinic I witnessed an experienced African American serial participant tell a Filipino immigrant, who was in his second study, his versions of the most common legends. Much of the newcomer's attention was focused on the amputation and skin graft tales. Shortly after their exchange I interviewed the immigrant participant. In response to my question about the types of studies he would be less inclined to do, he remarked:

I mean, it was just a story from another guy, one of the guys who didn't do the toe [study himself] ... I don't know if I believe him or not. Because he's telling something, maybe I believe the part right here [pointing to his skin], but the toe, what's the use, you know? What's the purpose of that toes, you know? [Laughs]

Nevertheless, contemporary legends like this one have the potential to be perceived as recommendations or guidance for those who are seeking higher paying studies, as research staff also reported receiving inquiries from participants who wanted to find amputation studies.

\section{The NASA study}

Whereas the origins of the flatliner and toe amputation studies are unknown, contemporary legends about a study conducted by NASA have a clear provenance. NASA's Johnson Space Center actually does conduct studies with healthy volunteers. ${ }^{5}$ While the healthy volunteers refer to the NASA study as one single ongoing study, the Space Flight Simulation Study, as it is formally called, consists of a series of studies designed to find countermeasures to protect astronauts' bodies from the deleterious effects of zero gravity. The study protocols vary, but most contain a period of bed rest, including tilt tests in which the beds are placed at slightly downward or upward angles. The length of the studies varies dramatically from several days to over 3 months continuously in bed. Compensation depends on the total length of the study, but one protocol lasting 115 days, including 90 of those days on bed rest, paid participants $\$ 17,000$ (Madrigal 2008).

As difficult as the actual NASA studies must be, the contemporary legend contains exaggerated details, so that the physical challenge of participating appears unendurable. For example, I heard variations of the legend that extended the bed rest period to a full year, added unpalatable dietary requirements, incorporated a solitary confinement to the experience and distorted the actual 5-15 degree tilt in the studies to a full upside-down position (both vertical and horizontal). At times, NASA disappeared from the tale (sometimes appearing as almost a misfired 'Nassau' or even 'NASCAR'). For example, a

\footnotetext{
${ }^{5}$ The official website can be accessed at NASA (n.d.).
} 
white serial participant in his late twenties brought up the version he heard (or remembered) when I asked him about the kinds of studies he would not want to participate in. He recalled:

I actually heard there was a study down in Houston, Texas. It was basically, what I heard was they were trying to give the patient - or the person that's volunteering muscular dystrophy. Basically keep them bedridden for 30 days straight, and I guess after a certain amount of time the human body, you know. I don't know what the details of it were or whatever, but that study seems like a heavy-duty one right there, you know.

I asked, 'Would you do that kind of study?' He pondered it for a moment and then replied:

Naw, I don't think so. That would be a tough one. I mean the compensation is pretty good. But like shew! I don't know, that's a pretty heavy duty one there.

In this instance, the erasure of NASA made the bed rest study even more difficult to understand, allowing the insertion of muscular dystrophy into the tale as a way to make sense of the known details.

The hyperbolic versions of the bed rest studies spark a lot of conversations among healthy volunteers. I frequently heard (and was asked) the question, 'Do you know about the NASA study?' Regardless of the answer, the person who posed the question would often then launch into his or her version of the tale, leading to discussions among participants about what they would or would not be able to handle and how the level of compensation would shape their perceptions of what would be tolerable. A few of the healthy volunteers I interviewed claimed to have actively pursued a spot in the NASA study, but either decided not to participate after learning the details or were disqualified because of a felony conviction on their record. One such healthy volunteer was an African American serial participant in his late thirties, who asked if I was familiar with the NASA study. I replied, 'The one that you're basically in bed for 90 days, right? Something like that?' He replied:

Yes, that study, but it's more to it than that. I was actually gonna screen for that study. I had an invitation to come down. The more I learned about it, I was like, 'No, I don't think I want to do that'... Because what they're actually doing is trying to simulate the astronaut's experience in space, so [you're] kinda gonna be in the bed for the duration, you know 3 months or whatever it was, but you'll be upside down. I was like, 'Upside down? ... For 3 months?' They said, 'Well, not the entire 3 months because every morning we're gonna get you up for 1 hour and spin you around in the centrifuge and then we're gonna put you back in the bed'.... I was like, man, it didn't sound too healthy. It didn't sound like something I wanted to go through. And then at the end of that you know, you have, what was it, maybe $\$ 18,000, \$ 20,000$, which would have been, you know, good enough to get you over the hump, but I don't know the type of damage that that would actually do to you long term. And I don't do well upside down at all.

Even among the small group of participants who claimed to have inside knowledge of the NASA study, the description they gave was more in the domain of contemporary legend. Perhaps the strenuousness of the actual study was enough for healthy volunteers to know that the study payment was not worth their time and participation but in order to justify 
passing on such a large amount of money, the details had to be exaggerated to a level where it could become more acceptable to refuse. The fascination with the NASA study coupled with the healthy volunteers' general lack of interest in participating in it could be interpreted as a concrete illustration of the boundary of what most serial participants are willing to do for income. It also signals an awareness, especially as seen through the last quote, of the potential long-term side effects of their participation in research.

\section{The dead participant}

There are surprisingly few stories that circulate about healthy volunteers who die as a result of their participation in a clinical drug trial. Perhaps these stories are simply too extreme for the participants to take much pleasure in telling them while they are enrolled in their own studies. In all the stories of this type I heard, the unexpected twist is that the death is always blamed on the participant himself or herself. Even when the contemporary legend is told in such a way that the cause of death is ambiguous, the healthy volunteers distance themselves from this extreme outcome by blaming the dead participant. The moral of this contemporary legend hinges on the importance of being honest with phase I researchers to protect one's health and life.

One story I heard seemed to be based on the 2004 suicide of Traci Johnson, a 19-year old healthy volunteer who hanged herself in a bathroom in the pharmaceutical company Lilly's testing facility after receiving multiple doses of duloxetine, an antidepressant (Harris 2004). In spite of the similarities with the Johnson case, none of the versions of this story I heard placed the healthy volunteer or the study at Lilly but instead inserted more familiar clinics. An African American serial participant in his forties gave me the following account:

I heard a suicidal case three years ago. Somebody took a antidepressant medication, and went home, and committed suicide... This was out here in [Eastern city], and I knew the guy too. Maybe something else was wrong with him. Because when you make out the consent forms, they tell you, 'Whatever's wrong with you, please be truthful and report it to us because the medication you're taking can have a certain effect on your body', especially the ones that mess with your central nervous system.

The framing that something was wrong with this story's participant is a familiar trope and was also used by Lilly and others during the Food and Drug Administration (FDA) investigations that occurred in the wake of Johnson's suicide. ${ }^{6}$

A different tale about the death of a participant was tied less to a specific clinical trial or type of drug and was instead infused with ethnic overtones. I heard this contemporary legend in the Southwest from several non-Hispanic whites. One such serial participant in his forties gave me his version of the incident:

Somebody died here [in this clinic]. She lied about her age, and she was Hispanic. They didn't check the ID then, and so she died from this place. She was too young,

\footnotetext{
${ }^{6}$ The FDA ruled that Johnson's suicide was not drug-related. In the intervening years there has been a growing amount of evidence that antidepressants like duloxetine cause suicide, especially in adolescents. It is possible that if the FDA were to make a determination about Johnson's death today, the agency would come to a different conclusion.
} 
she took the medicine, and it wasn't good at all .... So you really need to be honest before you try to get in here, no matter if you really need the money or not, because it will harm you. I mean, you have to be honest, telling the truth on your health history. That's important, right? You know, if you do that [lie], then you basically just cause a problem. You know, obviously, with that girl, she lied with her age and she didn't have the right ID, and she died here.

In this story, lying to the clinic staff is multifaceted. On one hand, there is the explicit accusation that she lied about her age and about any underlying conditions she might have had. On the other, there is the implicit critique that she also lied about her immigration status, using a false ID that the clinic failed to verify. My informant's rather unsympathetic framing of the victim herself can be situated in the context of ethnic tension that exists in the Southwestern USA, where immigrants are often seen as 'causing' problems. Regardless of the racist subtext, this type of contemporary legend communicates the notion that the healthy volunteers must be honest or suffer the consequences, including death.

\section{Productive functions of contemporary legends}

Stories like these about study participation are not just idle conversation among healthy volunteers. Sharing them may certainly be an excellent way to pass the time when confined to a phase I clinic but as narratives they perform even beyond building cohesion among participants. Contemporary legends are powerful devices because the narrators can adapt them to the situation, putting their own spin on the details while preserving the moral. In this way, these stories can be seen as having two functions. Firstly, contemporary legends provide the basis for coping with stigma through social comparisons. Participants can compare themselves to others who are more desperate or more indiscriminate about their enrolment in clinical trials. Secondly, they help create a gradient of risk that draws a line between acceptable and reckless study participation and, in the face of financial temptation, this can remind healthy volunteers to protect themselves from harm.

Given that US medical research is a stigmatised activity for many healthy volunteers, contemporary legends about clinical trials can be viewed as insertions of outsiders' perceptions of research onto the stories that insiders tell each other. As mentioned above, perceived stigma prohibits many healthy volunteers from discussing trial participation with others outside of the clinic. One white serial participant in his forties explained why he does not tell his family or friends about his participation in studies:

This is a very small percentage of people who are A) even thinking about doing it [enrolling in studies], and B) who are willing to do it. You go out on the street, and you tell people you're doing medical testing. They're like, 'Where's your third eye?' or 'Are you cutting off an arm?'

At the same time, the stigma experienced by the healthy volunteers is not simply that they participate in medical studies. Instead, it is seen as an implied admission of financial desperation. For example, an African American woman in her early twenties told me: 
I tend to be pretty secretive about the things that I do simply because everybody doesn't understand, you know. Everybody isn't the type of person who would be in a study for money.

Contemporary legends become the basis for downward social comparisons with fictional healthy volunteers who are more desperate than the teller or listener. ${ }^{7}$ Or, as explained by the Filipino immigrant in his fifties, the legends are compelling to healthy volunteers because 'they wanna make themselves feel like, "Oh, I'm not the worst one" .... Somebody's even worse than [me]'. This provides a mechanism to assert that money does not make them indiscriminating; there are studies they would refuse. Thus, it is possible for healthy volunteers to view their own decisions about clinical trials as reasonable ones in which they are not taking on extreme risks.

At the same time that healthy volunteers use contemporary legends to feel better about their participation in clinical trials, the moral conveyed by the stories simultaneously highlights a gradient of risk across clinical trials and reminds them to be vigilant in protecting themselves. Even though the content of the contemporary legends exaggerates the form the risks will take, part of the message is not to be blind to those risks because of the promised study compensation. An African American serial participant in his forties cleverly asserted this point, referencing the flatliner legend when talking about his own financial motivation to enrol in studies:

[The decision to participate] all boils down to dollars and cents ... I normally read the consent form and pretty much do something I wanna do, so that's how I figure out what [drug] I wanna take... 'Coz a study could pay you six grand, but they might wanna stop your heart beating or something. Nah, I don't think I'll do that, you know what I mean? So it's dollar and sense, have sense [Laughs].

Many participants, in fact, make fast and loose references to the contemporary legends when they talk about how money can influence people to make foolish decisions. Another African American serial participant in his forties told me about how he emphasises this point to other healthy volunteers:

I'll tell some of my friends, 'It's like when you call the recruiters, the only thing you hear is the compensation. Listen to what they're saying!' 'Oh, we're paying $\$ 3,500$ for this? ... We're gonna chop your head off'. Or, 'We're gonna put your feet in a basket'. And people are quick to say, 'Yeah'. [Laughs] Next time I see Joe Schmo, he has no head. 'Where's your feet at, man?' People say yes to anything. It's all about listening to what they actually tell you. The first thing you hear is [the compensation], ... your heart is pounding: 'God, let me see if I can get a $\$ 6,000$ study'.

\footnotetext{
${ }^{7}$ I am drawing on social identity theory here to explain how comparisons with others are used to form one's sense of self. Some scholars have differentiated between downward and upward comparisons to describe how these social comparisons operate. In the case of downward comparison, people use the case of an unfortunate other -whether real or imagined - to bolster positive perceptions of their own situation or position (see Locock and Brown 2010, Wills 1981).
} 
Contemporary legends provide healthy volunteers with an opportunity to rehearse decisions about potential risky studies from the likely (for example, lumbar punctures) to the improbable (for example, flatliners).

A second moral of the contemporary legends hinges on teaching healthy volunteers to be truthful and responsible in their interactions with phase I clinics. In effect, the stories responsibilise volunteers for ensuring their own wellbeing, while diminishing liability on the part of the clinics. The stories show that consequences are dire when individuals try to manipulate the clinics by lying about their health or failing to observe mandatory washout periods. ${ }^{8}$ In this way, contemporary legends may also have a positive effect on the healthy volunteers' behaviour. After hearing so many of these stories, an African American man in his thirties declared:

I don't wanna risk one drug interacting with another and stuff like that ... [The clinic] where I just came from, the guys I was staying with, they'd go back to back to back, and they do 'em [studies] around the country .... And you don't know what's gonna interact with what one day, and just your heart stops or something ... And so, I'm like, [sighs] I don't know if that is worth it. Doing all that is not worth it.

This is not to say that the contemporary legends are effective in persuading healthy volunteers always to be truthful about their study participation. Of course, many participants are focused on maximising how much money they can earn by enrolling in studies at multiple clinics, so they must be dishonest to qualify for the studies. Nonetheless, contemporary legends about dead volunteers can serve as a reminder to those participants that they are indeed taking risks.

\section{Conclusion}

In discussing the risks of USA phase I trials with healthy volunteers and researchers, both groups tend to frame these studies as low risk (for example, Kass et al. 2007, Sibille et al. 2006). Yet healthy volunteers enthusiastically circulate stories about dangerous and illegitimate clinical trials as part of their interaction with each other during the studies. Rather than understanding this phenomenon as a contradiction, it can instead be seen as a mechanism by which the healthy volunteers' discomfort with medical research can be explored through both humour and fear while creating an opportunity for downward social comparison in a stigmatised activity.

In the scholarly literature on contemporary legends, two common threads characterise many popular tales: vulnerability and distrust (Campion-Vincent 2002, Fine 1992, Turner 1993). Analysing contemporary legends about phase I studies in this light shows the residual anxieties that healthy volunteers have about participating in clinical trials. In a sense, the contemporary legends provide a residence for the stereotypes and fears of medical research that even insiders have difficulty rejecting. Whether or not the healthy volunteers believe the

\footnotetext{
${ }^{8}$ Phase I trials usually require a 30-day washout period between the last day of one study and the first day of the next. Clinics enforce washout periods for studies at their own clinics, but healthy volunteers can typically avoid this restriction on their participation by going to another clinic.
} 
legends, the stories confirm for them that the studies in which they are participating are not so dangerous. More than that, these stories are a medium to assert that there are limits to what they will do for money. In his analysis of contemporary legends about organ theft, Dingwall (2001) emphasised that sociologists should analyse not only the content of these narratives but also how they are communicated - who is telling them to whom and for what purpose. A logical extension of this directive is to investigate how contemporary legends produce effects on those who recount and listen to them. In this way, these narratives are not just stories, but tales that shape individuals' perceptions and behaviour, especially in the context of risky activities.

In this article I have illustrated how phase I contemporary legends have a productive function for healthy volunteers in the USA. It is unclear how the US phase I context differs from other countries in terms of which socioeconomic groups are frequent participants in these clinical trials. Clearly, the US history of the abuse and exploitation of disenfranchised minorities is an important - even if unspoken - backdrop to their participation in phase I clinical trials (see, Briggs 2002, Hornblum 1998, Reverby, 2009). At the same time, the USA has institutionalised policies to increase the participation of minorities and women in federally funded clinical research as a corrective to their historical exclusion in studies from which they could benefit (Epstein 2007). In the USA context of phase I trials, these incredible stories establish a gradient of risk regarding different types of procedures and studies, warn participants about the dangers of dishonesty about their trial history and emphasise that money is not more important than health and life. Contemporary legends, then, serve to remind individuals to protect themselves, rendering an uncertain risk visible for all healthy volunteers.

\section{Acknowledgments}

This research was supported by grant number 1R21CA131880 from the National Cancer Institute. Its contents are solely the responsibility of the author and do not necessarily represent the official views of the National Cancer Institute, National Institutes of Health. I am grateful for the assistance of Dulce Medina and Irma Beatriz Vega de Luna in conducting interviews with Spanish-speaking healthy volunteers.

\section{References}

Abadie, R. The Professional Guinea Pig: Big Pharma and the Risky World of Human Subjects. Durham, NC: Duke University Press; 2010.

Addams J. A modern devil-baby. American Journal of Sociology. 1914; 20(1):117-8.

Almeida L, Azevedo B, Nunes T, Vaz-da-Silva M, et al. Why healthy subjects volunteer for phase I studies and how they perceive their participation? European Journal of Clinical Pharmacology. 2007; 63(11):1085-94. [PubMed: 17891536]

Best J, Horiuchi GT. The razor blade in the apple: the social construction of urban legends. Social Problems. 1985; 32(5):488-99.

Briggs, L. Reproducing Empire: Race, Sex, Science, and US Imperialism in Puerto Rico. Berkeley, CA: University of California Press; 2002.

Brunvand, JH. The Vanishing Hitchhiker: American Urban Legends and Their Meanings. New York: Norton; 1981.

Brunvand, JH. The Choking Doberman: And Other Urban Legends. New York: Norton; 1984.

Campion-Vincent V. Organ theft narratives as medical and social critique. Journal of Folklore Research. 2002; 39(1):33-50. 
Cavens L, Ramael S. Cerebrospinal fluid sampling in phase 1 clinical trials: mind over matter? SGS Life Science|Technical, Bulletin. 2009; 30:1-5.

Corbie-Smith G, Thomas SB, Williams MV, Moody-Ayers S. Attitudes and beliefs of African Americans toward participation in medical research. Journal of General Internal Medicine. 1999; 14(9):537-46. [PubMed: 10491242]

Corrigan OP. 'First in man': the politics and ethics of women in clinical drug trials. Feminist Review. 2002; 72(1):40-52.

DiFonzo N, Bordia P. Rumor, gossip and urban legends. Diogenes. 2007; 54(1):19-35.

Dingwall R. Contemporary legends, rumours, and collective behaviour: some neglected resources for medical sociology? Sociology of Health \& Illness. 2001; 23(2):180-202.

Donohue J. A history of drug advertising: the evolving roles of consumers and consumer protection. Milbank Quarterly. 2006; 84(4):659-699. [PubMed: 17096638]

Elliott C. Guinea-pigging. The New Yorker. 2008 Jan 7.:36-41. [PubMed: 18265451]

Epstein, S. Inclusion: The Politics of Difference in Medical Research. Chicago: University of Chicago Press; 2007.

Fine, GA. Manufacturing Tales: Sex and Money in Contemporary Legends. Knoxville, TN: University of Tennessee Press; 1992.

Fisher, JA. Medical Research for Hire: The Political Economy of Pharmaceutical Clinical Trials. New Brunswick: Rutgers University Press; 2009.

Fisher JA, Kalbaugh CA. Challenging assumptions about minority participation in U.S. clinical research. American Journal of Public Health. 2011; 101(12):2217-22. [PubMed: 22021285]

Fox Tree JE, Weldon MS. Retelling urban legends. American Journal of Psychology. 2007; 120(3): 459-76. [PubMed: 17892088]

Harris G. Student, 19, in trial of new antidepressant commits suicide. New York Times. 2004 Feb 12.:A30.

Helms, R. Guinea Pig Zero: An Anthology of the Journal for Human Research Subjects. New Orleans: New Mouth for the Dirty South; 2005.

Hornblum, AM. Acres of Skin: Human Experiments at Holmesburg Prison. New York: Routledge; 1998.

Kass NE, Myers R, Fuchs EJ, Carson KA, et al. Balancing justice and autonomy in clinical research with healthy volunteers. Clinical Pharmacology and Therapeutics. 2007; 82(2):219-27. [PubMed: 17410122]

Locock L, Brown JB. 'All in the same boat'? Patient and carer attitudes to peer support and social comparison in motor neurone disease (MND). Social Science \& Medicine. 2010; 71(8):1498-505. [PubMed: 20719422]

Madrigal, A. [accessed 19 June 2014] NASA Offers \$5000 a month for you to lie in bed. Wired. 2008. Available at http://www.wired.com/2008/05/nasa-offers-500/

Moriarty E. Telling identity stories: the routinisation of racialisation of Irishness. Sociological Research Online. 2005; 10:3.

National Aeronautics and Space Administration. [last accessed 25 May 2014] Bed rest studies. n.d. available at https://bedreststudy.jsc.nasa.gov

Reverby, SM. Examining Tuskegee: The Infamous Syphilis Study and Its Legacy. Chapel Hill, NC: University of North Carolina Press; 2009.

Roche B, Neaigus A, Miller M. Street smarts and urban myths: women, sex work, and the role of storytelling in risk reduction and rationalization. Medical Anthropology Quarterly. 2005; 19(2): 149-70. [PubMed: 15974325]

Shavers-Hornaday VL, Lynch CF, Burmeister LF, Torner JC. Why are African Americans underrepresented in medical research studies? Impediments to participation. Ethnicity and Health. 1997; 2:1-2. 31-45.

Shibutani, T. Improvised News: a Sociological Study of Rumor. Ardent Media; 1966.

Sibille M, Donazzolo Y, Lecoz F, Krupka E. After the London tragedy, is it still possible to consider phase I is safe? British Journal of Clinical Pharmacology. 2006; 62(4):502-3. [PubMed: 16817849] 
Tishler CL, Bartholomae S. Repeat participation among normal healthy research volunteers: professional guinea pigs in clinical trials? Perspectives in Biology and Medicine. 2003; 46(4):50820. [PubMed: 14593220]

Turner, PA. I Heard It through the Grapevine: Rumor in African-American Culture. Berkeley, CA: University of California Press; 1993.

Victor JS. Moral panics and the social construction of deviant behavior: a theory and application to the case of ritual child abuse. Sociological Perspectives. 1998; 41(3):541-65.

Wills TA. Downward comparison principles in social psychology. Psychological Bulletin. 1981; 90(2): 245-71. 


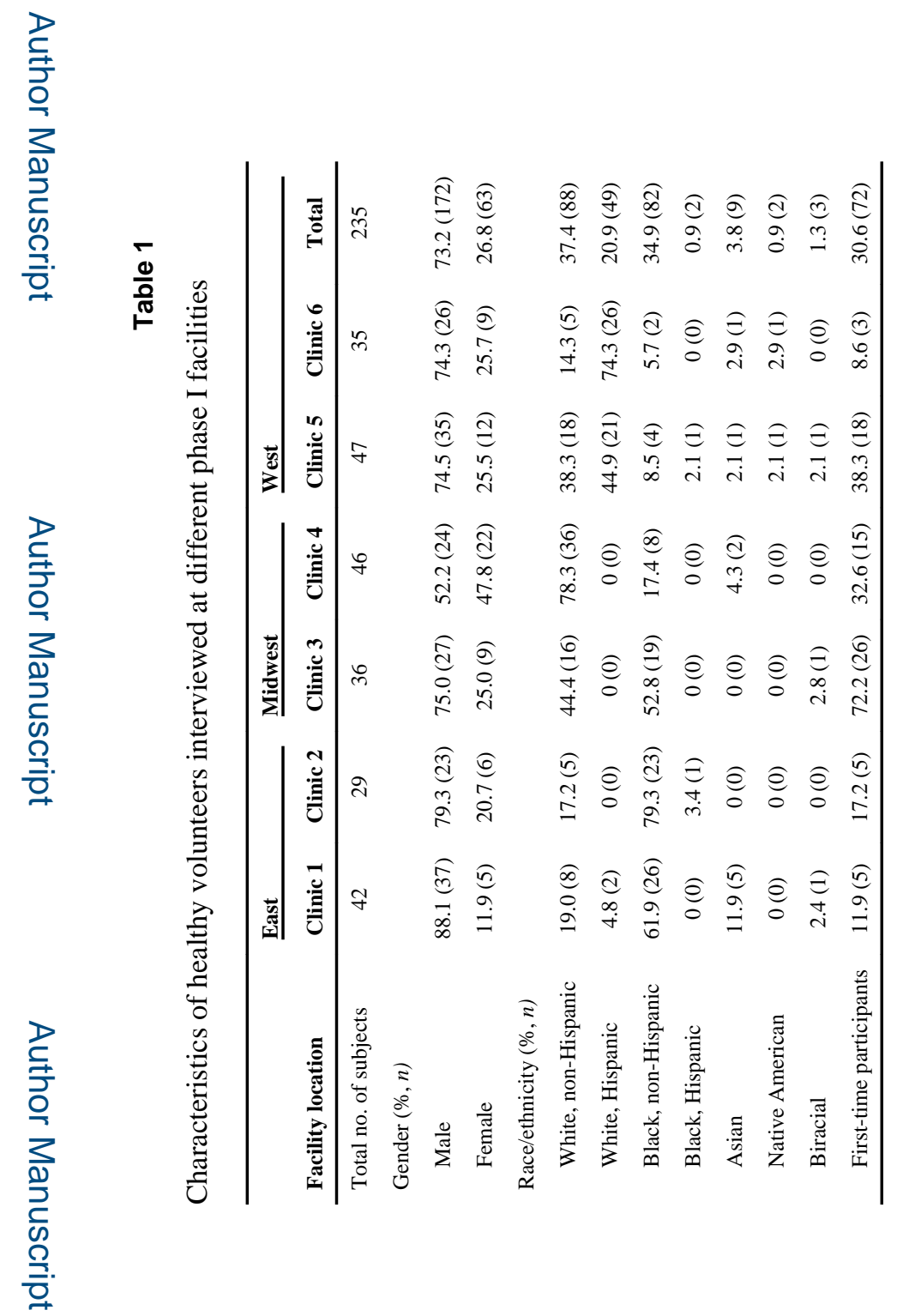

